\title{
A flexible robotic cell for in-process inspection of multi-pass welds
}

David I Lines, Yashar Javadi, Ehsan Mohseni, Momchil Vasilev, Charles N MacLeod, Carmelo Mineo, Randika W Vithanage, Zhen Qiu, Rastislav Zimermann, Charalampos Loukas, Euan Foster, S Gareth Pierce, Anthony Gachagan

Centre for Ultrasonic Engineering (CUE), Department of Electronic \& Electrical Engineering, University of Strathclyde,Glasgow, G1 1RD, UK

Telephone - 0141-444 7404

Telefax - 0141-552 2487

david.lines@strath.ac.uk

\section{Abstract}

Welds are currently only inspected after all the passes are complete, and after allowing sufficient time for any hydrogen cracking to develop, typically over several days. Any defects introduced between passes are therefore unreported until fully buried, greatly complicating re-work and also delaying early corrections to the weld process parameters. In-process inspection can provide early intervention but involves many challenges, including operation at high temperatures with significant gradients affecting acoustic velocities and hence beam directions. Reflections from the incomplete parts of the weld would also be flagged as lack of fusion defects, requiring the region of interest to adapt as the weld is built up. The collaborative SIMPLE (IIngle Manufacturing PLatform Environment) project addresses these challenges by incorporating robotic inspection within a robotic TIG welding cell. This has been accomplished, initially with commercial off-the-shelf ultrasonic phased arrays, but flexibly enough to adapt to future developments with higher temperature solutions. The welding and inspection robots operate autonomously. The former can introduce deliberate defects to validate the latter which uses $5 \mathrm{MHz} 64$ element phased arrays on 
high temperature wedges to generate sector scans after each weld pass. Results are presented, confirming the challenges have been addressed and demonstrating the feasibility of this approach.

\section{Introduction}

There are international standards [1, 2, 3] for ultrasonic Non-Destructive Testing (NDT) of welds.

These require a period, typically of several days, between completion of the weld and inspection to allow for any hydrogen cracking to develop. Any defects will therefore remain undetected until this final inspection, and rework of a buried defect will involve removing all of the weld passes above the defect. All of this greatly increases manufacturing cost and makes it highly desirable to detect defects after each weld pass, so that the final inspection verifies that it is defect-free.

The nuclear industry is being called upon to play a major role in providing a resilient, carbon-free power generation network. This will require significant reductions in manufacturing cost and the SIMPLE project, funded under the UK's Nuclear Innovation Programme (NIP), aims to reduce this by $50 \%$. For example, the 40 weld passes on an $80 \mathrm{~mm}$ thick $2 \mathrm{~m}$ diameter pressure vessel currently requires 40 sets of cooling cycles, inspections and then re-heat, which can take a total of 120 days

[4]. If this in-process inspection could take place at elevated temperatures, much of this wasted time could be avoided and so provide a means to achieve the required cost reduction.

The welding environment is very hostile in both temperature and electromagnetic interference. Most ultrasound NDT transducers will not tolerate temperatures above $60^{\circ} \mathrm{C}$ and so alternative approaches will be required to offer the cost benefits. The ultrasound instrumentation is frequently operating at high gain, close to the thermal noise limits, and so suppression and filtering techniques 
will be needed to reject interference from welders, robot motors and the general industrial environment.

The imaging algorithms rely on knowing the speed of sound but, since this drops with increasing temperature, surface temperature measurements will be required to provide appropriate compensation. When inspecting close to the weld head, there will be large temperature gradients within the parent material and so the sound will no longer travel in straight lines. This will cause geometrical distortions as well as potentially affecting the amplitude threshold for reporting an echo as a defect.

Once the weld is complete, the ultrasound inspection detects a Lack of Fusion (LOF) defect as an unexpected specular refection located at the original metal profile surface. There will be many such reflections from un-welded surfaces, progressively reducing in extent as more weld passes are added. Image analysis on the part-completed weld will need to mask these false-positive responses, whilst still responding correctly to defects within the completed part. Some form of adaptive masking will therefore be required.

\section{Addressing the technical challenges}

\subsection{Robotic welding cell}

The University of Strathclyde (UofS) has a pair of flexible robotic welding cells which are currently capable of Tungsten Inert Gas (TIG) and Metal Inert Gas (MIG) welding, the TIG cell being used for the SIMPLE project. The harsh and restricted environment means that a second robot is needed for the in-process ultrasonic inspection. 


\subsubsection{Robotic cell setup}

- The weld robot is a 6-axis Kuka KR5 ARC HW with a KRC2 controller. As well as the TIG weld head, the weld robot has a laser profiler and a video camera to monitor the weld pool.

- Pre-heating can be applied via resistance blankets driven from a DHC65150 power unit from Thermal Hire Ltd.

- The inspection robot, used to place the ultrasound array sensor head, is a 6-axis Kuka KR6 R900 with a KRC4 controller.

Figure 1 shows the robotic cell with welding and inspection robots.

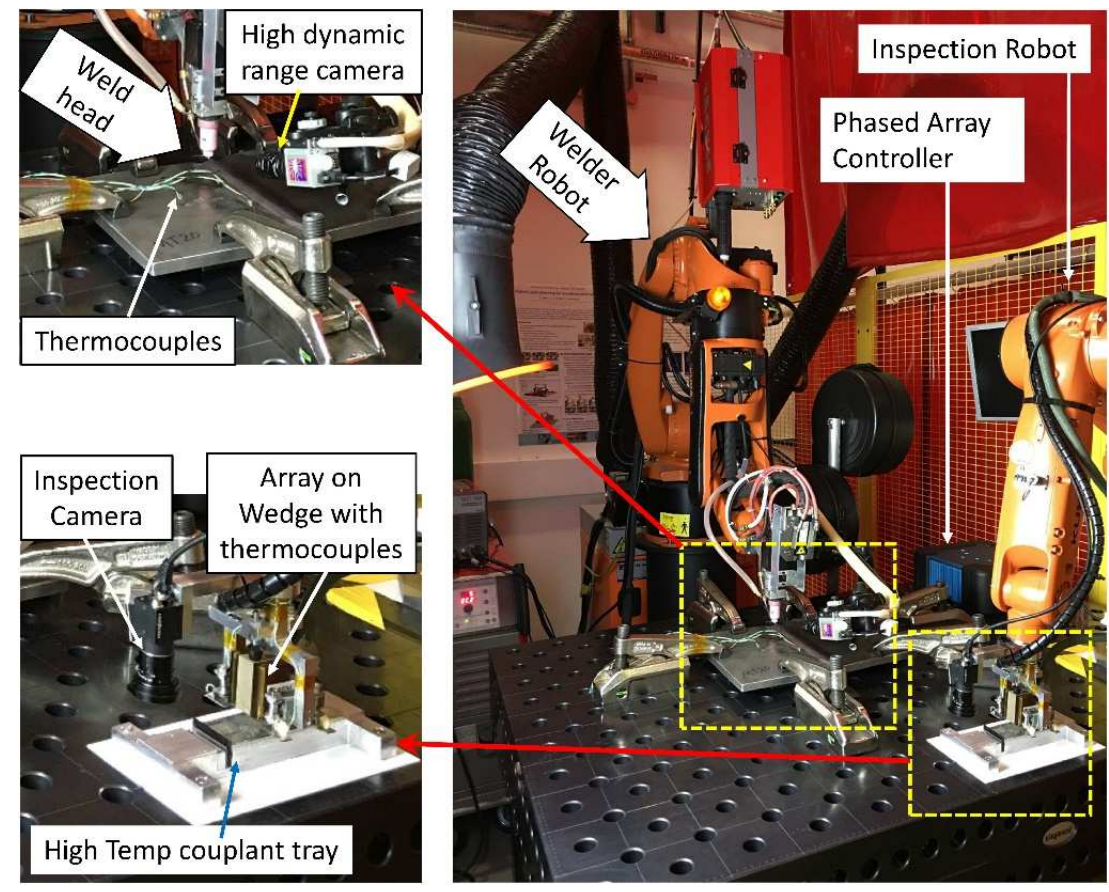

Figure 1. In-process inspection of multi-pass robotic welding

Real-time synchronizing and control for the Kuka Robots and Welding Control unit is via LabVIEW ${ }^{\mathrm{TM}}$ code (LabVIEW 2017-32bit) running on a cRIO-9038 from National Instruments. The cRIO controller 
also has a 16-channel thermocouple interface for monitoring temperatures from both the array head and for any thermocouples welded onto the top surface of the weld parent material. The controller is responsible for monitoring welding parameters (voltage, current, etc.) and logging these with the thermocouple measurements, profiler data, video and ultrasonic data during the welding and inspection. The inspection robot can be left running over an extended period after welding (typically 3 days) for monitoring the development of hydrogen-cracking.

\subsubsection{Creating known weld defects}

The UofS team has developed a range of techniques for generating repeatable known defects during welding, including lack of fusion, inclusions, porosity and hydrogen-cracking. Comparing ultrasonic detection results during welding and after cooling with destructive testing $[5,6,7](1)(2)$ confirms the validity of the inspection process.

\subsection{Ultrasonic Inspection}

\subsubsection{Tolerance of sensor to elevated temperatures}

The majority of ultrasound NDT transducers use the piezo-electric effect for both transmission and reception, with PZT being most commonly used piezo material because of its sensitivity. The Curie temperature is dependent on PZT type, ranging from $71^{\circ} \mathrm{C}$ to over $250^{\circ} \mathrm{C}$ [8]. When combined with epoxies in composite transducers, the maximum operating temperature without degradation is around $60^{\circ} \mathrm{C}$. Acoustic wedges between transducer and target material are used to generate the required shear wave inspection angles and these blocks can also be used to insulate the piezomaterial from the temperatures of the material, allowing inspection to be done at $150^{\circ} \mathrm{C}$ [9]. UofS is developing a high temperature ultrasonic roller probe, but this would not be available within the 
timescale of the project's current phase. However, the robotic end effector has been designed with it in mind for future use.

Other higher temperature piezo materials are in use for high temperature inspection, particularly for process monitoring in the oil and gas industries. They are usually permanently fixed, with solid deformable couplant films compressed between transducer and target. Currently there are no commercially available arrays in these materials that can be scanned over the surface. Non-contact approaches that can inspect high temperature materials include Electromagnetic Acoustic Transducers (EMATs) and Laser Ultrasound systems, and were considered as they are also couplantfree. Both show lower sensitivity than their piezo contact alternatives and have less control over the inspection angle. This is likely to change as they become more widely used, and the robotic cell would be an ideal research environment to evaluate them in the future.

\subsubsection{Ultrasonic array and high temperature wedge}

Commercially available ultrasound arrays are recommended to operate below $60^{\circ} \mathrm{C}$. For weld inspection, they are mounted on acoustic wedges so that they only generate angled shear waves for inspecting the weld. The wedge therefore also acts as a temperature barrier and so operation on inspection surfaces above $60^{\circ} \mathrm{C}$ is possible. Olympus has developed a range of elevated temperature wedges manufactured using an amorphous thermoplastic polyetherimide resin called ULTEM ${ }^{\mathrm{TM}}$, and they can operate at surface temperatures up to $150^{\circ} \mathrm{C}[9]$.

While ULTEM's thermal insulating properties are used to protect the probe, heat dissipation is also important to enable a longer inspection period. When the ambient temperature is $25^{\circ} \mathrm{C}$, the wedge can by kept in contact with a $150^{\circ} \mathrm{C}$ surface for a maximum of 10 minutes. It takes an additional 10 
minutes for the probe and wedge to cool. As the temperature increases from $24{ }^{\circ} \mathrm{C}$ to $150{ }^{\circ} \mathrm{C}$, the ultrasonic velocity of the ULTEM wedge material drops from $2470 \mathrm{~m} / \mathrm{s}$ to $2270 \mathrm{~m} / \mathrm{s}$, while the shear wave velocity in steel drops from $3240 \mathrm{~m} / \mathrm{s}$ to $3103 \mathrm{~m} / \mathrm{s}$. The appropriate values for the operational temperature need to be used when calculating the appropriate electronic steering and focusing delays to transmit and receive beams from the desired locations.

For this phase of work, the only currently available solution is the array and wedge that can tolerate $150^{\circ} \mathrm{C}$ surface temperatures. The combined assembly is mounted in a frame attached to the inspection robot end effector. The mounting includes sprung-loaded thermocouples to contact the surface before the wedge. The thermal distribution output from weld-modelling software is used to predict safe surface temperature for placing the wedge, but the thermocouples distributed around the mounting provide a further level of safety. The outputs from these thermocouples can also be used for a 4-point measure of temperature to assist in any calculation of thermal gradients. Figure 2 (left) shows the ultrasound sensor head comprising array, wedge, thermocouples and mounting. These can be combined in pairs, as shown in Figure $\mathbf{2}$ (right), on the robotic end effector to inspect from both sides of the weld, and in pitch-catch mode through the weld.
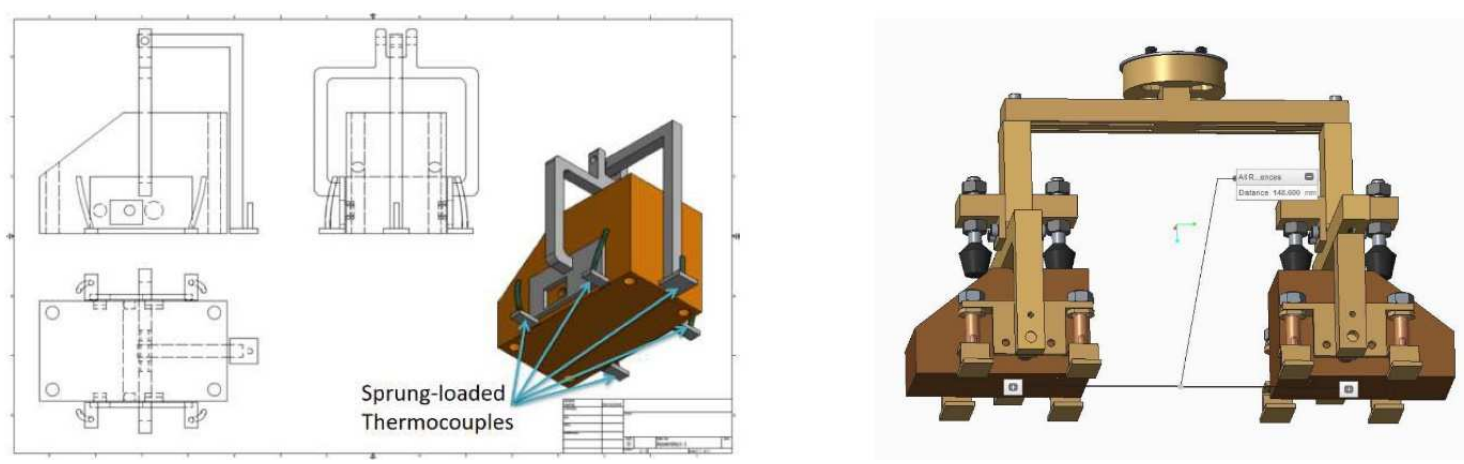

Figure 2. Ultrasound sensor head - acoustic wedge with sprung thermocouples (left) and twin head (right) 


\subsection{Tolerance to noise and interference}

The welder and the robots have the potential to generate strong electromagnetic interference that can affect the quality of the data being collected by the ultrasound phased array system. Initial tests showed that the interference generated when the arc strikes was sufficient to switch off the phased array unit and also reset the controller module used to collect the thermocouple outputs. The latter was resolved by modifying the code to detect when it was subject to a reset condition, and to reinitialise all the relevant parameters. Optimising the location of the Phased Array Controller (PAC), and the earthing/shielding arrangement of the array and controller avoided this being reset by the arc striking. There was still electrical interference on the output of the PAC from both welder and robots, but this was outside the bandwidth of interest in the ultrasonic data and was successfully removed by high-pass filtering of the data.

\subsection{Suppression of false positive indications}

\subsubsection{Staircase reference block}

NDT standards require the inspection equipment to be setup on calibration blocks, the acceptance levels set as a threshold, and any echo exceeding the threshold to be flagged as a potential indication. The material used for the calibration block will be the same as (or as close as practicable to) the material to be inspected. For inspection at elevated temperatures, the ideal is to have the calibration block at the same temperature, otherwise appropriate compensation (such as a change to the acoustic velocity) will be needed. In addition to the calibration block, it is common practice for there to be a reference block as close as possible to the inspection geometry and containing features that should be detected. Typically, both the calibration block and the reference block would be 
inspected before and after the shift to confirm the validity of any inspection results that occurred during the shift.

In-process inspection will require the amplitude threshold to be constrained to within a region of interest, which progressively expands as the weld passes are laid down. A "staircase" test block, containing the full range of weld cross section profiles (all stages from none to fully-welded) in the same block, is therefore an ideal reference.

\subsubsection{Specification of Staircase reference block}

The material properties and the profile geometry of the pre-welded Staircase reference block are detailed in Table 1 and the properties of each weld pass are given in Table $\mathbf{2}$.

Table 1. Material properties and pre-welded geometry

\begin{tabular}{|l|l|}
\hline Property & Value \\
\hline Material & Steel (S275) \\
\hline Material length & $300 \mathrm{~mm}$ \\
\hline Material thickness & $15 \mathrm{~mm}$ \\
\hline Weld profile & $90 \mathrm{deg} \mathrm{V}$ \\
\hline Root face & $1.5 \mathrm{~mm}$ \\
\hline Root gap & $2.1 \mathrm{~mm}$ \\
\hline
\end{tabular}

Table 2. Staircase reference block weld pass definition

\begin{tabular}{|l|l|l|l|}
\hline Pass & Length & Pass Sequence & Thickness \\
\hline Step 1 (Root pass) & $270 \mathrm{~mm}$ (0 to $270 \mathrm{~mm})$ & 1 & $3.2 \mathrm{~mm}$ (to $3.2 \mathrm{~mm}$ ) \\
\hline Step 2 (Hot Pass) & $230 \mathrm{~mm}$ (0 to $230 \mathrm{~mm}$ ) & 2 & $1.5 \mathrm{~mm}$ (to $4.7 \mathrm{~mm}$ ) \\
\hline Step 3 & $190 \mathrm{~mm}$ (5 to $195 \mathrm{~mm})$ & 3,4 & $2.44 \mathrm{~mm}$ (to $7.14 \mathrm{~mm}$ ) \\
\hline
\end{tabular}




\begin{tabular}{|l|l|l|l|}
\hline Step 4 & $152 \mathrm{~mm}$ (8 to $160 \mathrm{~mm})$ & $5,7,6$ & $2.26 \mathrm{~mm}$ (to $9.4 \mathrm{~mm}$ ) \\
\hline Step 5 & $117 \mathrm{~mm}$ (8 to $125 \mathrm{~mm})$ & $8,10,11,9$ & $3.46 \mathrm{~mm}$ (to $12.86 \mathrm{~mm}$ ) \\
\hline Step 6 & $80 \mathrm{~mm}(10$ to $90 \mathrm{~mm})$ & $13,15,16,14,12$ & $2.14 \mathrm{~mm}$ (to $15 \mathrm{~mm}$ ) \\
\hline Step 7 (Cap) & $45 \mathrm{~mm}(10$ to $55 \mathrm{~mm})$ & $19,20,21,18,17$ & Up to $4.3 \mathrm{~mm}$ (to $19.3 \mathrm{~mm}$ ) \\
\hline
\end{tabular}

Figure 3 shows the top surface of the completed Staircase reference block, with the array on high temperature wedge aligned with the mark to show the inspection position.

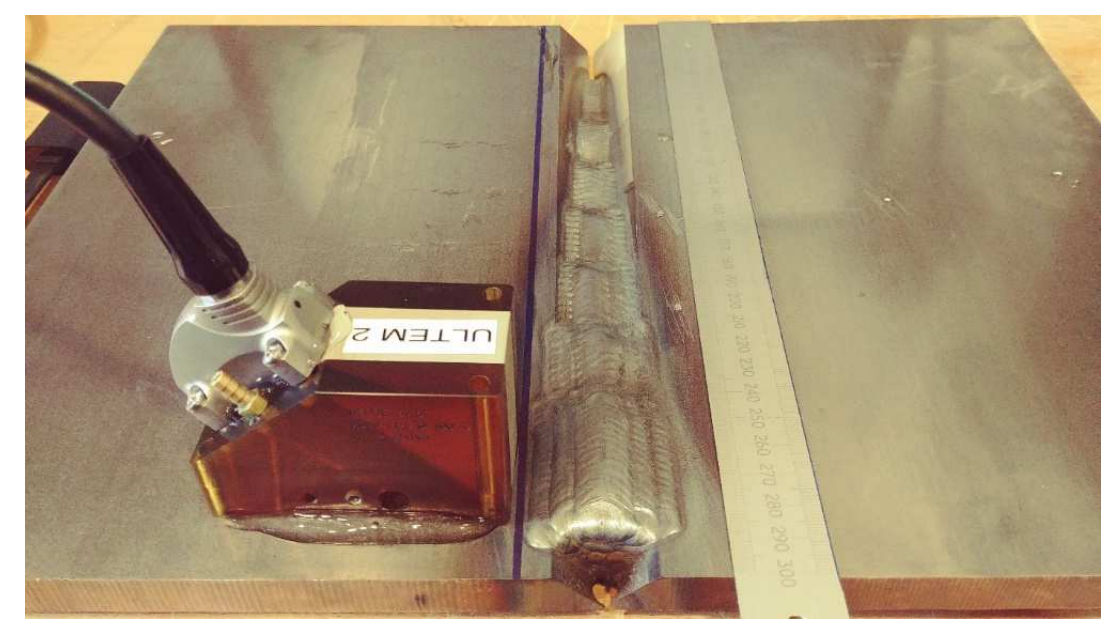

Figure 3. Top view of completed staircase test block with alignment mark for wedge positioning

\subsubsection{Results from Staircase reference block}

The equipment used to inspect of the Staircase reference block is listed in Table $\mathbf{3}$.

Table 3. Equipment used to inspect Staircase reference block

\begin{tabular}{|l|l|l|}
\hline Equipment & Serial No. & Comments \\
\hline $\begin{array}{l}\text { Peak NDT LTPA Phased } \\
\text { Array controller }\end{array}$ & 18015 & $\begin{array}{l}\text { 64 channel transmit / 64 channel receive / 64 } \\
\text { element. } \\
\text { Data collected using Peak NDT's “ArrayGen" software } \\
\text { and Peak NDT's LabVIEW version. }\end{array}$ \\
\hline $\begin{array}{l}\text { Olympus 5MHz Array } \\
\text { 5L64-32X10-A32-P-2.5-HY }\end{array}$ & T3728 & $\begin{array}{l}\text { 5MHz 64 element phased array with 0.5mm element } \\
\text { pitch. }\end{array}$ \\
\hline
\end{tabular}




\begin{tabular}{|l|l|l|}
\hline $\begin{array}{l}\text { Olympus High } \\
\text { Temperature Acoustic } \\
\text { Wedge }\end{array}$ & $\begin{array}{l}550 \text { acoustic wedge fabricated from ULTEM high } \\
\text { temperature acrylic with wedge angle of } 37.6 \circ \text { and } \\
\text { acoustic velocity at room temperature of } 2470 \mathrm{~m} / \mathrm{s} \\
\text { and } 2340 \mathrm{~m} / \mathrm{s} \text { at } 100^{\circ} \mathrm{C} .\end{array}$ \\
\hline $\begin{array}{l}\text { Olympus High Temp } \\
\text { Couplant H-2 (Q7700002) }\end{array}$ & $-23^{\circ} \mathrm{C}$ to $398^{\circ} \mathrm{C}$ \\
\hline
\end{tabular}

The different regions of the weld are inspected using beam angles matched to the typical defects. Multiple reflections (skips) off the top and bottom faces are required for small-angled beams to reach the bevelled surface, whilst the root can be interrogated with a direct high-angle beam. The ray paths, shown in Table 4, illustrate this combination of angle and number of skips needed to inspect each colour-coded region of the weld.

Table 4. Ray paths, for varying number of beam skips, used to inspect different regions (colourcoded) of the weld cross section

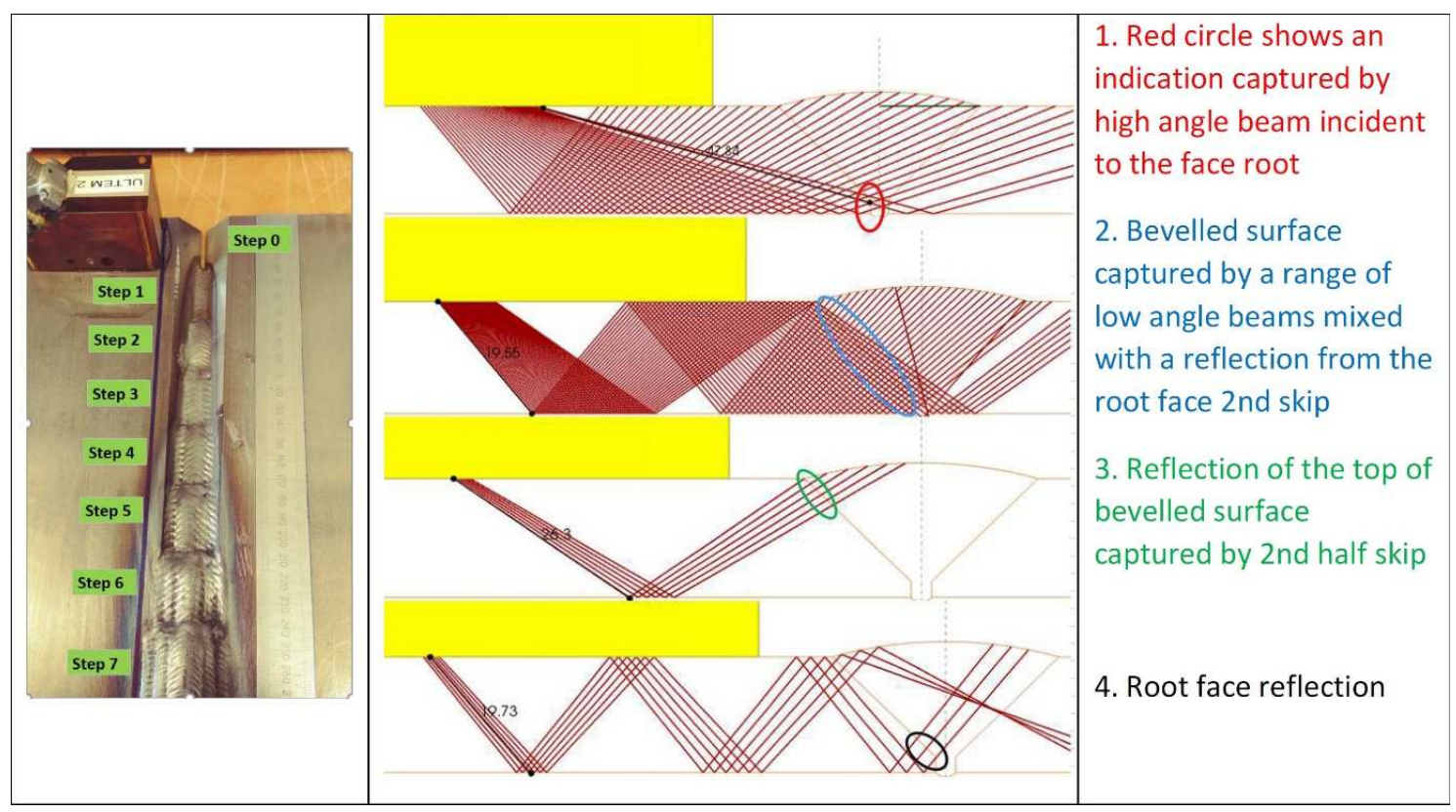


Table 5 shows the weld cross section geometry for each step of the Staircase reference block, showing the weld passes for each case, and the matching sector scan image.

Table 5. Weld cross section and image for each step of the Staircase reference

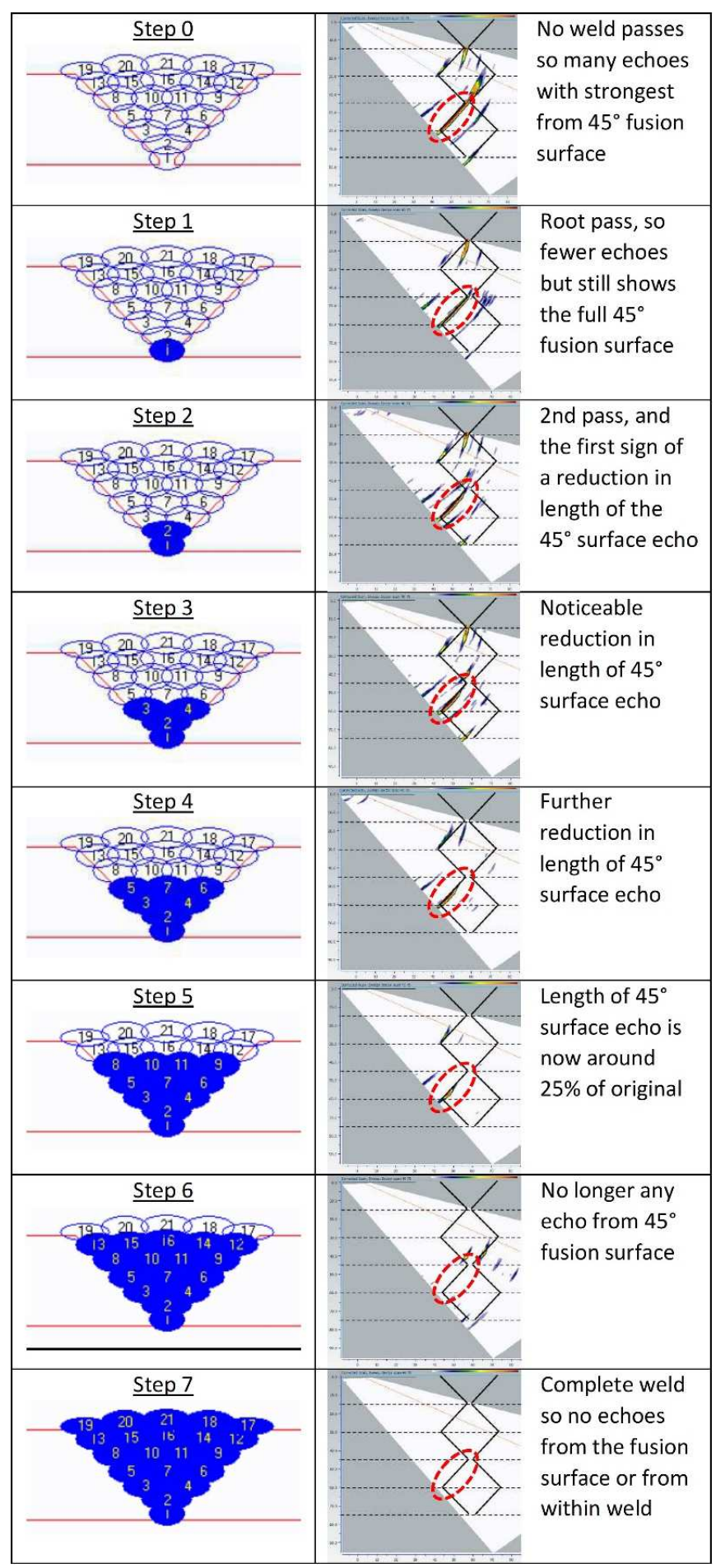




\subsection{Temperature-related geometric distortions}

Although the wedge protects the array from the degradations that occur above its maximum operating temperature of $60^{\circ} \mathrm{C}$, image distortions will arise from:

- Reduction in the acoustic velocity in the wedge from increasing temperature via the inspection surface. If the whole wedge is at a uniform elevated temperature, it would increase the apparent range to an echo due to the increased transit time in the wedge. The change in refractive index would increase the refracted beam angle and further distort the image. The uniform temperature is easily measured and allows appropriate compensation to correct for the distortion.

- The wedge material is chosen to provide a thermal barrier to protect the array, so the low thermal conductivity will give rise to large temperature gradients in the wedge, and these will bend the beams within the wedge.

- Both the above will change with time, from the point where the array wedge makes contact with the inspection surface. The low conductivity means the temperature gradient will be in a narrow strip along the inspection interface.

- Reduction in the acoustic velocity in the material due to the increase in temperature from welding (and any pre-heating). If the acoustic path from wedge to weld is at the same elevated temperature, the larger transit time would produce an increase in the apparent echo range. The refractive index change reduces the refracted beam angle and also produces a geometrical distortion. As with the wedge, a uniform temperature in the target material can be measured and appropriately compensated.

- If inspecting soon after the weld pass, there will be large temperature gradients in the material and these will bend the beams within the material. 
Majidnia and Nageswaran presented the results of TWI's ultrasound beam modelling simulations, using the CIVA modelling package, of the effects of temperature gradients across the weld on the echo amplitude and location [10]. They reported a limited amplitude change, around -1 or $-2 \mathrm{~dB}$, with the main effect being a positional change of up to $5 \mathrm{~mm}$. This would be sufficient to affect the positioning of the Region of Interest (ROI) to mask the false-positives from the interfaces yet to be welded. Our view is that the echoes from the interfaces yet to be welded, can be used to provide a reference to correct for most of the geometrical distortion. The position of the ROI mask can be moved to align with the distorted position of these reference echoes, and the location of real defects within the welded part can be made relative to the interface.

\subsubsection{Modelling of temperature gradients}

To verify the results of the CIVA modelling performed by TWI, Finite Element Analysis (FEA) simulations were performed using PZflex. Potentially it can also look at temperature gradients within the wedge. The model confirms the TWI prediction that there is a marginal change in echo amplitude but a geometrical distortion of up to $5 \mathrm{~mm}$.

\subsubsection{Results of elevated temperature imaging on the Staircase reference block}

Data collection and imaging were performed at room temperature and elevated temperatures to assess the geometrical distortions. In particular, it was important to see whether the distortion is primarily a shift, allowing the corrections to be done by aligning the echoes from the un-welded interface with their expected location.

The following approach was adopted (all temperatures measured with handheld probe): 
- Array+wedge placed on Step 2 of the Staircase block using high temperature couplant, and reference scan acquired at room temperature $\left(21.6^{\circ} \mathrm{C}\right)$.

- Location was marked so that could subsequently be replaced at the same position.

- Array+wedge removed and pre-heater blanket raised temperature of the Staircase block to $160^{\circ} \mathrm{C}$, and then blanket removed allowing the block to cool.

- When temperature reached $150^{\circ} \mathrm{C}$, array+wedge were clamped back on the block. Images were acquired at intervals, recording the temperature.

- After 6 minutes, the array and wedge were removed, to cool for 10 minutes.

- The wedge was then replaced, the elapsed timer reset, and more data acquired.

Figure 4 shows the results representing the two extremes of temperature. In both cases, the temperature distribution was almost uniform throughout the sample and, because the elevated temperature frame was acquired a few seconds after placing the array and wedge, there is likely to be little temperature gradient within the wedge.
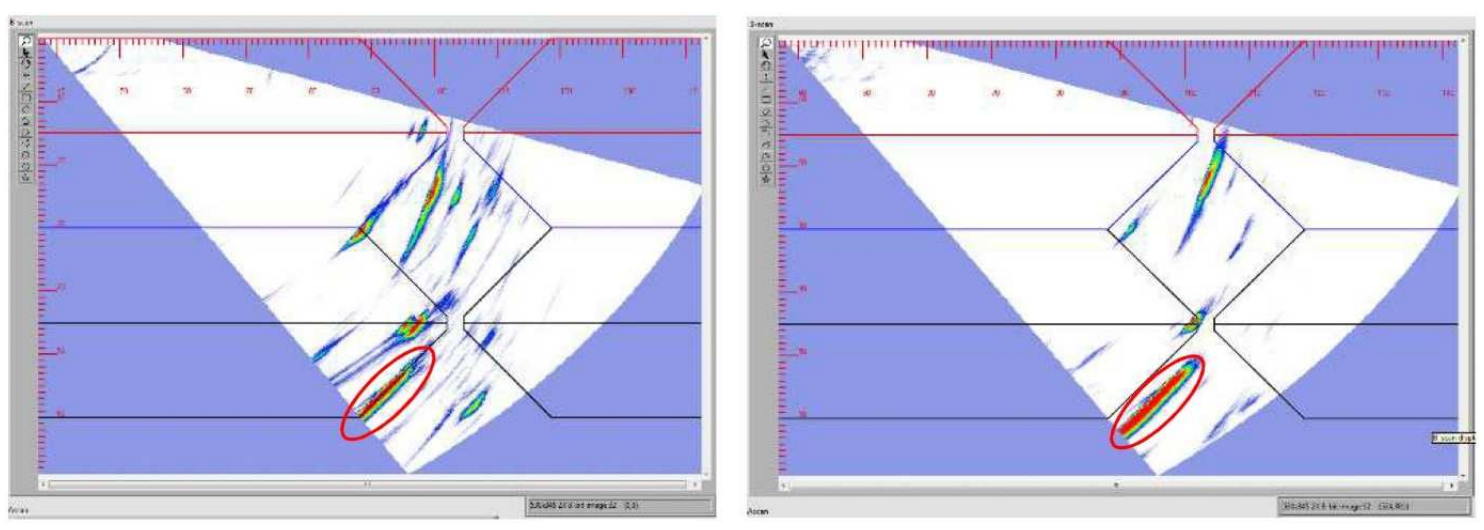

Figure 4. Comparison of reference image at $21.6^{\circ} \mathrm{C}$ (left) and image at $150^{\circ} \mathrm{C}$ (right) showing shift in reference feature by around $3 \mathrm{~mm}$ 
The images were acquired using velocities matching those at room temperature (wedge velocity $=2470 \mathrm{~m} / \mathrm{s}$ and steel shear wave velocity of $3240 \mathrm{~m} / \mathrm{s})$. The propagation distance to the reflector is around $85 \mathrm{~mm}$. At $150^{\circ} \mathrm{C}$, the shear wave velocity drops to $3103 \mathrm{~m} / \mathrm{s}$ and so the displayed range (at the original velocity) would increase to about $88.7 \mathrm{~mm}$. This suggests that correcting for the change in velocity would remove much of the misalignment and so geometrical errors can be tolerated.

\subsection{Modification of imaging/analysis for in-process inspection of multi-pass welding}

The focal delay laws and the scan conversion into an image rely upon defined acoustic velocities, which can be adjusted to reflect the values at elevated temperatures. Temperature gradients, in either wedge or target, will bend the beams and the amount can be derived from verified weld simulations. There is therefore a need for a scan-conversion algorithm that handles beam bending. This has been implemented in a new LabVIEW library (.Ivlib) which is still fast enough ( $\sim 5 \mathrm{~ms}$ for a typical high-resolution image) for the most extreme data acquisition and display rates. The resulting image is then imported into the LabVIEW Vision module, where overlays can be used to indicate the pre-weld geometries, assisting analysis of the echoes, as shown in Figure $\mathbf{5}$.

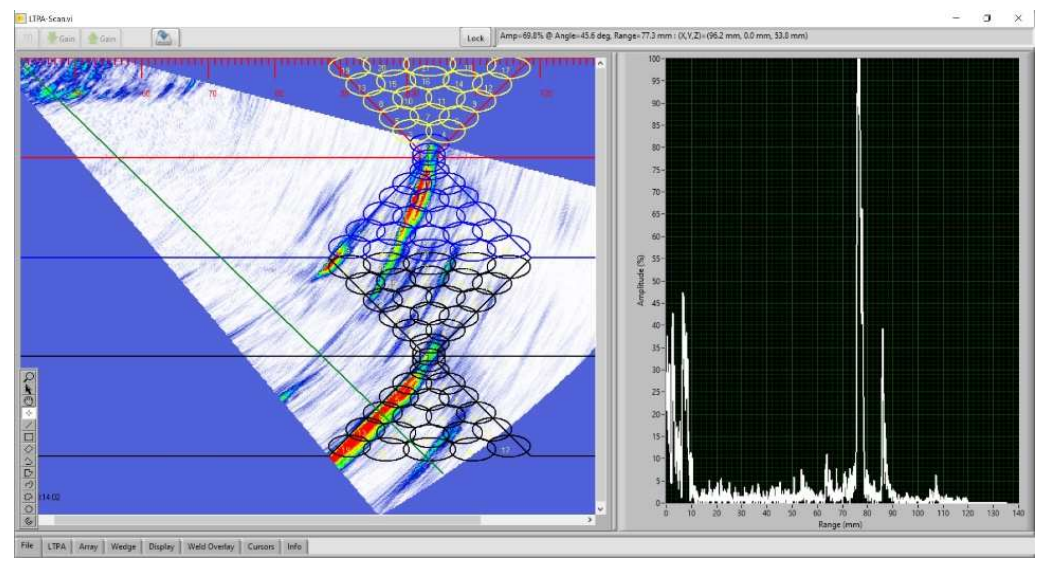

Figure 5. Scan-converted image + overlay of pre-weld geometry and weld passes 
The LabVIEW Vision module also supports localised Regions of Interest (ROIs) which can be used to mask the false-positives that will result from the un-welded interfaces of a part-filled weld.

- Figure 6A illustrates the standard inspection case, where a complete weld is inspected and a LOF defect, shown in blue, is identified and correctly reported.

- Figure 6B shows the in-process inspection where the weld is good, but the remaining bevelled surface produces LOF-like echoes, shown in red, which would be false-positives if reported.

- Figure $6 \mathrm{C}$ shows the in-process inspection where the weld is poor. There are both true-positive LOF indications in blue, which must be reported, and false-positives from the incomplete weld surface in red, which must not.

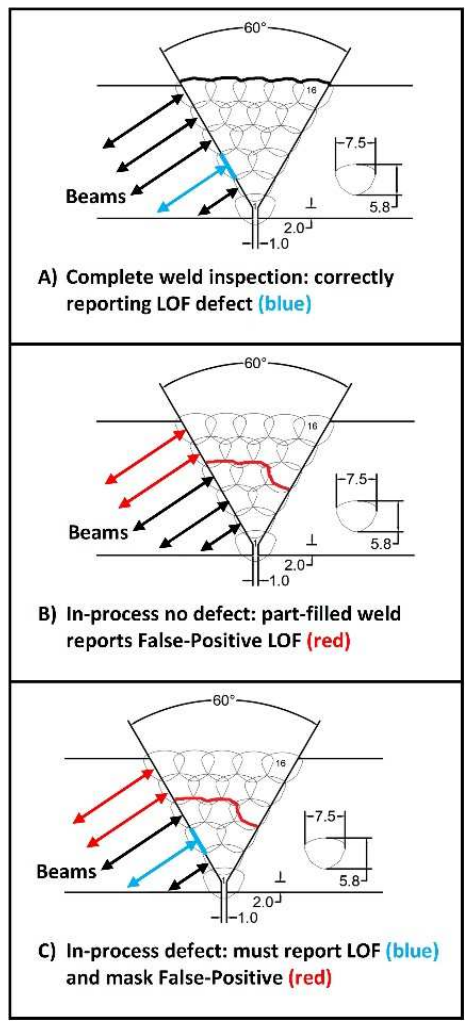

Figure 6. Inspection configurations 


\section{Conclusions}

The paper has explained why in-process inspection is highly desirable to reduce costs, and identified the technical challenges. The hostile environment demands automated inspection and the dualrobot cell satisfies this requirement, yet remains flexible enough to adapt to future advances with high temperature ultrasound sensors. The weld process can be manipulated to generate known defects, and the full logging of parameters allows for potential data fusion and analysis. The ultrasonic instrumentation has been optimised to operate during welding and robot movements without compromising signal integrity.

Finite Element Analysis was used to predict the extent of the geometrical distortion resulting from the thermal gradients, and the imaging software has been modified to handle curved ultrasonic beams. A Staircase reference block was manufactured to assess how best to mask the False-Positive echoes from the parts of the weld profile yet to be filled, and has been used to compare images acquired at room temperature and at $150^{\circ} \mathrm{C}$. The geometrical shift was as expected and reinforces the plan to use these unwanted echoes as a known reference point for correcting for any geometrical distortion.

This work has demonstrated the viability of in-process ultrasonic inspection, initially leading to time and cost saving resulting in a final defect-free final inspection, but also gathering data in support of future in-process inspection standards.

\section{Acknowledgements}


The authors would like to thank:

- $\quad$ The department for Business, Energy and Industrial Strategy (BEIS) for sponsoring this research as part of the Nuclear AMRC's SIMPLE project - part of the BEIS Nuclear Innovation Programme.

- The Engineering and Physical Sciences Research Council (EPSRC) for their funding of parallel feed-in projects ( EP/L022125/1 UK RESEARCH CENTRE IN NON-DESTRUCTIVE EVALUATION (RCNDE) 2014-2020 core Project Acoustic Based Characterisation of Arc Fusion Parameters ( $A B C$ of $A R C), E P / N 018427 / 1$ Autonomous Inspection in Manufacturing \& Remanufacturing (AIMaReM) and EP/R004889/1 Delivering Enhanced Through-Life Nuclear Asset Management project ANRC 12-1 Advanced Ultrasonic inspection and Monitoring for Challenging Industrial Welding Applications.

- The rest of the team at the University of Strathclyde's Centre for Ultrasonic Engineering (CUE) and Peak NDT for their assistance on this work.

\section{References}

1. Continuous monitoring of an intentionally-manufactured crack using an automated welding and in-process inspection system. Javadi, Yashar, et al. June, s.I. : Journal of Materials and Design, 2020, Vol. 191.

2. Investigating the effect of residual stress on hydrogen cracking in multi-pass robotic welding through process compatible non-destructive testing. Javadi, Yashar, et al. April, s.l. : Journal of Manufacturing Processes, 2020.

3. BS EN ISO 17640:2010. Non-destructive testing of welds - Ultrasonic testing - Techniques, testing levels, and assessment (ISO 17640:2010). Non-destructive testing of welds - Ultrasonic testing - Techniques, testing levels, and assessment (ISO 17640:2010). s.I. : BSI Standards Publication, 2010. BS EN ISO 17640:2010.

4. BS EN ISO 13588:2012. Non-destructive testing of welds - Ultrasonic testing - Use of automated phased array technology (ISO 13588:2012). Non-destructive testing of welds Ultrasonic testing - Use of automated phased array technology (ISO 13588:2012). s.I. : BSI Standards Publication, 2012. BS EN ISO 13588:2012. 
5. BS EN ISO 19285:2017. Non-destructive testing of welds - Phased array ultrasonic testing (PAUT) - Acceptance levels (ISO 19285:2017). BS EN ISO 19285:2017. Non-destructive testing of welds - Phased array ultrasonic testing (PAUT) - Acceptance levels (ISO 19285:2017). s.I. : BSI Standards Publication, 2017. BS EN ISO 19285:2017.

6. InFORM - Intelligent Fixtures for Optimised and Radical Manufacture. Cook, B P. Sheffield : s.n., 2-3 July 2019.

7. Hooker, M W. Properties of PZT-Based Piezoelectric Ceramics between -150 and $250^{\circ} \mathrm{C}$. NASA/CR-1998-208708. [Online] September 1998.

https://ntrs.nasa.gov/archive/nasa/casi.ntrs.nasa.gov/19980236888.pdf.

8. Ultrasonic phased array wedge for inspecting high-temperature parts up to $150^{\circ} \mathrm{C}$. Olympus Industrial Resources - Applications Notes. [Online] [Cited: June 10, 2019.] https://www.olympusims.com/en/applications/ultrasonic-phased-array-wedge-for-inspecting-high-temperature-partsup-to-150c.

9. Intentional weld defect process : from manufacturing by robotic welding machine to inspection using TFM phased array. Javadi, $Y$, et al. Burlington, USA : 45th Annual Review of Progress in Quantitative Nondestructive Evaluation, 2018.

10. In-Process Inspection of Multi-Pass Robotic Welding. Javadi, Y, et al. Portland, OR, USA : Proceedings of QNDE, July 2019.

11. Ultrasonic phased array inspection of the fully-automated multi-pass weld with an intentionally embedded tungsten rod. Javadi, Y, et al. Telford, UK : Proceedings of NDT2019, September 2019.

12. Modelling of high-temperature ultrasonic inspection for in-process inspection of electron beam welding. Nageswaran, C and Majidnia, S. Nottingham, UK : Proceedings of NDT2018, September 2018. 